2 Detection of Iodomethane using Luminescent Lead Halide

4

5 Wenping Yin ${ }^{\dagger, 1,2}$, Hanchen Li ${ }^{\dagger, 1,2}$, Anthony S. R. Chesman ${ }^{1,3,4}$, Ben Tadgell ${ }^{1,5}$, Andrew D. Scully ${ }^{1,3}$,

6 Mingchao Wang ${ }^{2}$, Wenchao Huang ${ }^{2}$, Christopher R. McNeill ${ }^{2}$, Wallace W. H. Wong ${ }^{1,5}$, Nikhil V.

8

9

10

11

12

13

14

15

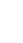

$$
\text { Medhekar }^{2} \text {, Paul Mulvaney }{ }^{1,5} \text {, Jacek J. Jasieniak }{ }^{*, 1,2}
$$

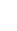

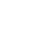

${ }^{1}$ Australian Research Council Centre of Excellence in Exciton Science, Australia

${ }^{2}$ Department of Materials Science and Engineering, Monash University, VIC, 3168 Clayton, Australia ${ }^{3}$ CSIRO Manufacturing, Clayton, VIC, 3168 Clayton, Australia ${ }^{4}$ Melbourne Centre for Nanofabrication, VIC, 3168 Clayton, Australia ${ }^{5}$ School of Chemistry, The University of Melbourne, VIC, 3010 Parkville, Australia

\title{
Perovskite Nanocrystals
}




\section{Abstract}

2

The extensive use of iodomethane $\left(\mathrm{CH}_{3} \mathrm{I}\right)$ as a pesticide has drawn serious concern due to its potential biological and atmospheric impact, yet there is currently no effective on-site monitoring method for determining iodomethane concentration. Here, we introduce a highly sensitive and selective photoluminescence (PL)-based $\mathrm{CH}_{3} \mathrm{I}$ sensor, that relies on changes in the optical properties of cesium lead bromide $\left(\mathrm{CsPbBr}_{3}\right)$ inorganic perovskite nanocrystals arising from halide exchange. $\mathrm{CH}_{3} \mathrm{I}$ alone does not react efficiently with $\mathrm{Cs}_{\mathrm{PbBr}}$. However, after the pre-exposure of $\mathrm{CH}_{3} \mathrm{I}$ to oleylamine a rapid alkylation reaction occurs, forming alkyl ammonium iodide species that undergo facile halide exchange with the perovskite nanocrystals. The extent of the halide exchange is directly dependent on the $\mathrm{CH}_{3} \mathrm{I}$ concentration, with the PL emission of the $\mathrm{CsPBBr}_{3}$ nanocrystals exhibiting a concomitant linear redshift of more than 150 nm upon the addition of $100 \mathrm{ppbv}$ to $10,000 \mathrm{ppbv}$ of $\mathrm{CH}_{3} \mathrm{I}$, and with a fast response time of ca. $5 \mathrm{~s}$. This is the most sensitive approach to the detection of $\mathrm{CH}_{3} \mathrm{I}$ using a low-cost and portable approach reported to date, with the limit of detection (LOD) being $30 \pm 10 \mathrm{ppbv}$, and according to simulations, could be improved even further. These PL changes are found to be independent of the intermediate morphology of the nanocrystals and operate effectively over a practical temperature range of $15-35^{\circ} \mathrm{C}$. The selectivity of the reaction mechanism is governed by the dramatic difference in the rate of the alkylation between $\mathrm{CH}_{3} \mathrm{I}$ and oleylamine compared with other organoiodine analogues. Furthermore, the facile transduction mechanism provides scope for the development of a portable and low-cost sensor system suitable for both visual and instrumental readout. 
Introduction

2

The high reactivity of iodomethane (methyl iodide, $\mathrm{CH}_{3} \mathrm{I}$ ) confers both its potential utility and hazard in human applications. Since its approval for use as a pesticide by the United States Environmental Protection Agency in 2007 it has gained popularity as a fumigant for insect and fungus control ${ }^{1,2}$. However, there has also been strong academic scrutiny and public concern regarding its latent toxicity ${ }^{3,4}$ due to the possible mutagenic and carcinogenic effects arising from its high reactivity with biological nucleophiles after prolonged exposure ${ }^{5}$. Furthermore, $\mathrm{CH}_{3} \mathrm{I}$ can release its constituent iodine atoms via photodecomposition, which can subsequently react with atmospheric ozone to produce the monoxide radical, IO, leading to ozone-depletion in the troposphere and stratosphere ${ }^{6,7}$. Because of the high reactivity of $\mathrm{CH}_{3} \mathrm{I}$ and the difficulties of detection due to its odourless and colourless nature, a rapid, on-site method for monitoring $\mathrm{CH}_{3} \mathrm{I}$ has remained elusive.

Traditionally, $\mathrm{CH}_{3} \mathrm{I}$ detection relies on sophisticated instrumentation, such as gas chromatography coupled with mass spectroscopy (GC-MS) or flame ionization detector based methods (GC-FID) $)^{7-10}$. However, these techniques are restricted to the laboratory environment and require complex sample preparation. An alternative approach involves the use of more affordable and portable spectroscopic methods. For example, a limit of detection (LOD) for $\mathrm{CH}_{3} \mathrm{I}$ of $5.3 \mathrm{ppt}\left(10^{-15}\right)$ was achieved using resonance fluorescence detection of atomic iodine $(170-190 \mathrm{~nm})^{6}$, although the utility of this system was greatly reduced by its low selectivity. To enhance selectivity, the strong methylating ability of $\mathrm{CH}_{3} \mathrm{I}$ (iodide as a leaving group) compared with other iodine containing species has also been exploited. Organic and organometallic luminescent probes, such as cyclometallated iridium (III) complexes ${ }^{11}$ and pyridine-based pi-conjugated oligomers/polymers ${ }^{2}, 5,12,13$, change their optical properties in response to iodomethane. However, the reactions proceed over minutes to hours, leading to 
1 lower sensitivity ( $>100 \mathrm{ppb}$ ) and long measurement times. It is, therefore, critical to develop a

2 reliable method for more rapid, selective, and sensitive detection of $\mathrm{CH}_{3} \mathrm{I}$.

The luminescence characteristics of cuboid lead halide perovskite nanocrystals (PNCs) are known to be highly dependent on the nature of the halide anion in the crystal lattice ${ }^{14}$. Consequently, perovskites are potential spectroscopic probes for the iodide component of $\mathrm{CH}_{3} \mathrm{I}$. Cuboid PNCs have the chemical formula $\mathrm{ABX}_{3}$, where $\mathrm{A}$ is a monovalent inorganic or organic cation such as cesium $\left(\mathrm{Cs}^{+}\right)$, methylammonium $\left(\mathrm{MA}^{+}\right)$or formamidinium $\left(\mathrm{FA}^{+}\right), \mathrm{B}$ is a divalent metal ion (usually $\mathrm{Pb}^{2+}$ or $\mathrm{Sn}^{2+}$ ), and $\mathrm{X}$ is a halide anion, typically chloride $\left(\mathrm{Cl}^{-}\right)$, bromide $\left(\mathrm{Br}^{-}\right)$, or iodide $\left(\mathrm{I}^{-}\right)$. The optoelectronic properties of PNCs are comparable, or even superior, to conventional group II-VI or III-V semiconductor quantum dots. In addition, they are cheaper and easier to synthesize and do not require a shell layer to passivate their surfaces owing to their remarkable defect tolerance ${ }^{15}$. In a landmark study in 2014, Schmidt et al. reported the synthesis of $\mathrm{MAPbBr}_{3}$ PNCs demonstrating a PL quantum efficiency (PLQE) of approximately $20 \%{ }^{16}$. Subsequently, synthetic improvements and an expansion of the compositional range to include various combinations of $\mathrm{Cs}^{+}, \mathrm{FA}^{+}$and modified $\mathrm{X}^{-}$, yielded PNCs with vastly improved PLQEs approaching $100 \%{ }^{17}$. An exciting property of PNCs is that their highly ionic nature allows their optoelectronic properties to be readily engineered post-synthesis through facile and rapid ion-exchange ${ }^{18}$. Through such exchange processes, tunable emission wavelengths throughout the entire visible spectrum (390-780 nm) with near-unity PLQE can be achieved ${ }^{17}$. While this tunability is typically exploited for optoelectronic applications, here we utilise the ability of such PNCs to undergo rapid halide exchange as a signal transduction method to detect $\mathrm{CH}_{3} \mathrm{I}$.

For this purpose, we focus on using highly photoluminescent inorganic cesium lead bromide $\left(\mathrm{CsPbBr}_{3}\right)$ PNC dispersions that are designed to efficiently interact with $\mathrm{CH}_{3} \mathrm{I}$. This is facilitated through the PNCs' native surface ligand, oleylamine (OLA), which acts as a 
1 sacrificial nucleophile in a reaction with $\mathrm{CH}_{3} \mathrm{I}$ that releases the iodide. Subsequent halide

2 exchange of the bromide in the PNCs with iodide results in a rapid change in the optical

3 properties. This mechanism allows for the detection of $\mathrm{CH}_{3} \mathrm{I}$ across a broad concentration range

$4 \quad(30 \pm 10 \mathrm{ppbv}$ to $20,000 \mathrm{ppbv})$, with a response time of ca. $5 \mathrm{~s}$. Moreover, the large differences

5 in the rate of nucleophilic substitution reaction between various organoiodines and OLA

6 provides for a high degree of selectivity of the $\mathrm{PNC}$-based indicator for $\mathrm{CH}_{3} \mathrm{I}$. First principles

7 density functional theory (DFT) calculations further confirm that stronger chemical

8 interactions of the resulting oleylammonium iodide compared to $\mathrm{CH}_{3} \mathrm{I}$ with the $\mathrm{PNC}$ surface,

9 also facilitates the high sensitivity of the indicator from a thermodynamic viewpoint.

\section{Results and Discussion}

The underlying premise of utilising $\mathrm{CsPbBr}_{3}$ PNCs for the detection of $\mathrm{CH}_{3} \mathrm{I}$ is based on the ability of PNCs to undergo halide exchange, which dramatically alters their spectroscopic characteristics ${ }^{14}$. However, the direct exposure of $\mathrm{CsPbBr}_{3} \mathrm{PNCs}_{\text {in toluene to }}$ $\mathrm{CH}_{3} \mathrm{I}$ solutions did not cause any discernible change in PL properties over modest timescales (Error! Reference source not found.a, Fig. S1a). This demonstrates that efficient halide exchange in perovskites only occurs between ionic halide species and not with covalentlybound iodine species ${ }^{19}$. To activate the sensor, we exploited the well-known methylation reaction between organoiodines $\left(\mathrm{R}_{3} \mathrm{C}: \mathrm{I}\right)$ and appropriate nucleophiles $(\mathrm{Nu}:)$ to generate $\left[\mathrm{R}_{3} \mathrm{C}: \mathrm{Nu}\right]^{+}$and free iodide $\left(\mathrm{I}^{-}\right)^{20}$. In this work, OLA was a convenient choice as the nucleophile given its use in the synthesis of the $\mathrm{CsPbBr}_{3} \mathrm{PNCs}$ :

$$
\mathrm{R}_{3} \mathrm{C}: \mathrm{I}+\mathrm{C}_{18} \mathrm{H}_{35} \mathrm{~N}: \mathrm{H}_{2} \rightarrow\left[\mathrm{R}_{3} \mathrm{C}: \mathrm{NH}_{2} \mathrm{C}_{18} \mathrm{H}_{35}\right]^{+}+\mathrm{I:}^{-}
$$


$1 \quad \mathrm{An}_{\mathrm{N}} 2$ reaction between $\mathrm{CH}_{3} \mathrm{I}$ and an excess of OLA initially yields $\mathrm{N}$-methyl oleylammonium

2 iodide (eq.1), while further reaction with additional OLA yields N-methyl oleylamine and an

3 oleylammonium iodide salt, as illustrated in Error! Reference source not found. $\mathbf{b}^{20,21}$. A

4 subsequent methylation reaction with $\mathrm{CH}_{3} \mathrm{I}$ is also possible, resulting in the formation of N,N-

5 dimethyl oleylamine and additional oleylammonium iodide. Evidence supporting this reaction

6 mechanism was obtained for a model OLA analogue, dodecylamine, using NMR and GC-MS

7 measurements (Fig. S2, S3, see Supporting Information for additional details).

The use of OLA in the synthesis of PNCs in the present work results in the concomitant

9 formation of its quarternized ammonium form when an acid is also present in the reaction

10 medium, such as oleic acid ${ }^{22}$. The OLA and/or oleylammonium salts subsequently passivate

11 the surface of the nanocrystals ${ }^{23}$. The addition of excess OLA into the PNC dispersions did not cause any changes in their PL emission wavelength (Error! Reference source not found.a, S1b).

In contrast, when a toluene solution of $\mathrm{CH}_{3} \mathrm{I}$ and $\mathrm{OLA}\left(\mathrm{CH}_{3} \mathrm{I}: \mathrm{OLA}=1: 3\right.$ molar ratio $)$ was added to a dispersion of the PNCs, significant changes in the optical properties of the PNCs were observed (Fig. 1b-dError! Reference source not found.). The red-shift in the UV-visible absorption and PL emission spectra upon $\mathrm{CH}_{3} \mathrm{I}$ addition is the result of a decrease in the optical bandgap of the cuboid nanocrystals, which is direct evidence for the exchange of bromide with iodide in the lattice of the $\mathrm{CsPbBr}_{3} \mathrm{PNCs}^{24}$. Notably, the dramatic redshift in PL is linearly correlated with the $\mathrm{CH}_{3} \mathrm{I}$ concentration in the range 100 ppbv to 10,000 ppbv (Fig. 1Error! Reference source not found.d), and is sufficiently large to provide a practical visual response according to the CIE chart shown in Fig. 1Error! Reference source not found.e. Further inspection of the absorption spectra for samples with $\mathrm{CH}_{3} \mathrm{I}$ concentrations above $3,000 \mathrm{ppbv}$ show an increased absorption at shorter wavelengths, which suggests the possible concurrent formation of 2D Ruddlesden-Popper perovskite (2DRP) nanosheets ${ }^{25}$. The generalized chemical formula for 2DRP nanosheets is given by $\mathrm{L}_{2}\left[\mathrm{ABX}_{3}\right]_{\mathrm{n}-1} \mathrm{BX}_{4}$, where $\mathrm{L}$ represents a 
1 monovalent organic cation that is usually larger than $\mathrm{A}^{+}$and the value of $n$ is the number of

2 metal halide layers. The UV-visible absorption energy profile (Fig. 1Error! Reference source

3 not found.c) and other results discussed below are consistent with the 2DRP nanosheets with

$4 \mathrm{n}=1$ and $\mathrm{n}=2^{26}$.
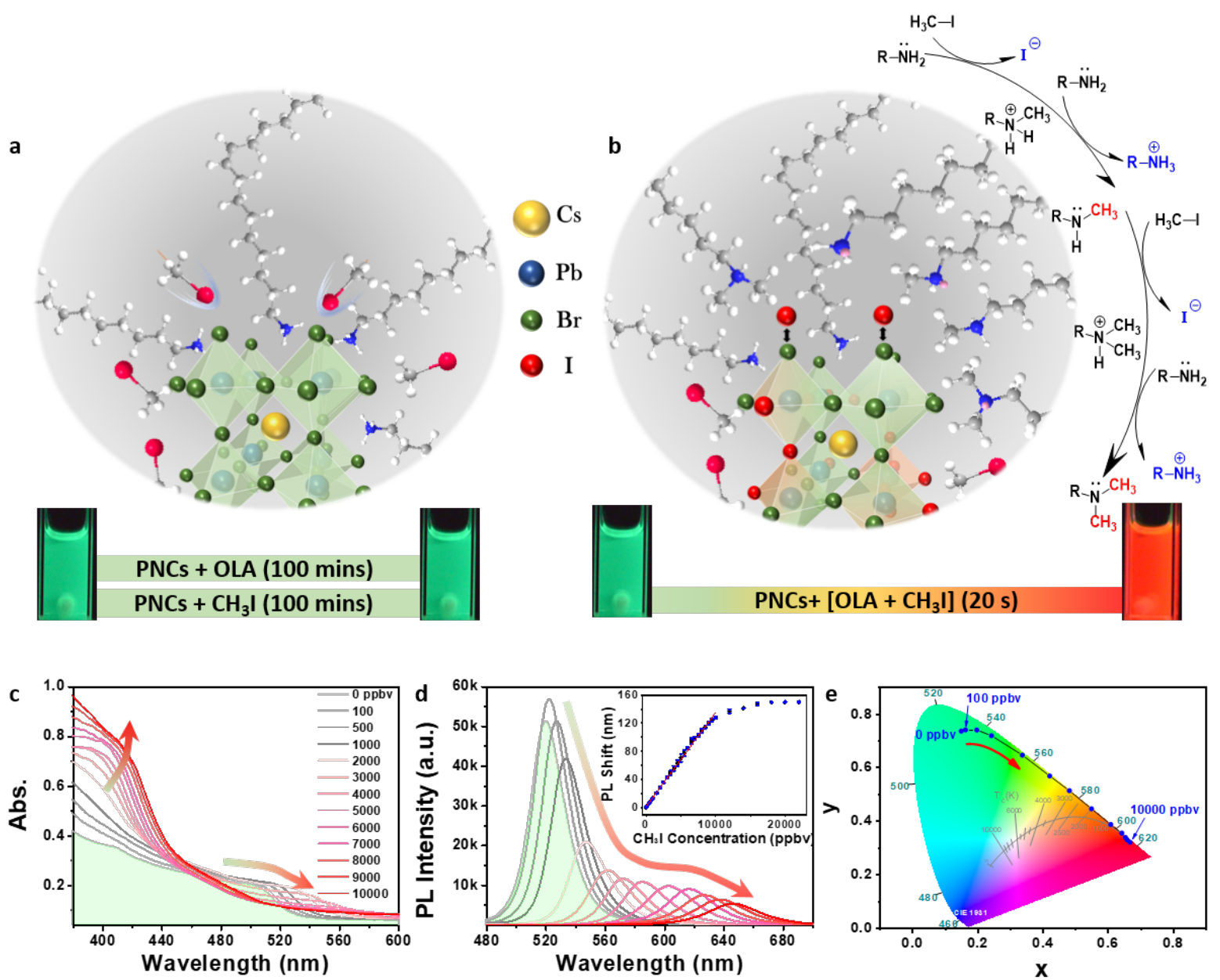

6

7

8

9

Fig. 1 | Reaction mechanisms and spectroscopic response of $\mathrm{CsPbBr}_{3}$ perovskite nanocrystals (PNCs) to $\mathbf{C H}_{3}$ I. a, Oleylamine (OLA, $\left.0.96 \mathrm{mM}\right)$ or $\mathrm{CH}_{3} \mathrm{I}(20,000$ ppbv solution) were introduced separately into PNC dispersions in toluene. The emission images under $365 \mathrm{~nm}$ UV light were recorded after 100 mins, showing no change in emission. b, OLA pre-treated $\mathrm{CH}_{3} \mathrm{I}$ solutions $\left(\mathrm{CH}_{3} \mathrm{I}\right.$ concentration: 20,000 ppbv) was added to a PNC dispersion in toluene, with the emission colour under $365 \mathrm{~nm} \mathrm{UV}$ light before and $20 \mathrm{~s}$ after addition. The hypothesised reaction mechanism, where $\mathrm{CH}_{3} \mathrm{I}$ induces the methylation of OLA by an $\mathrm{S}_{\mathrm{N}} 2$ mechanism and stops at dimethyl analogue formation. c, UV-visible 
1 absorption spectra of PNCs exposed to varying amounts of $\mathrm{CH}_{3} \mathrm{I}$. d, Emission spectra of $\mathrm{CsPbBr} \mathrm{PNCs}_{3}$

2 as a function of the amount of added $\mathrm{CH}_{3} \mathrm{I}$. (Insert: redshift of PNCs PL emission as a function of $\mathrm{CH}_{3} \mathrm{I}$

3 concentration. Linear fitting of results from $100 \mathrm{ppbv}$ to $10,000 \mathrm{ppbv}$ shown as a red line with $\mathrm{R}^{2}=$ 0.997). e, CIE chart converted from the PL spectra of PNCs exposed to varying amounts of $\mathrm{CH}_{3} \mathrm{I}$. (Note: Spectra in $\mathbf{c}$, d, were recorded $20 \mathrm{~s}$ after $\mathrm{CH}_{3} \mathrm{I}$ addition at room temperature to ensure the reaction was complete).

To correlate these spectroscopic changes with the underlying structural transformations in the PNCs, transmission electron microscopy (TEM) and X-ray scattering measurements were conducted. TEM images of the as-synthesized PNCs reveal a well-defined cuboid morphology, with an average size of $11 \mathrm{~nm}$ (Fig. 2a, S4). For $\mathrm{CH}_{3} \mathrm{I}$ concentrations up to 3,000 ppbv, high-resolution TEM (HRTEM) diffraction patterns indicate the progressive expansion of the (100) lattice spacing in the cuboid $\mathrm{CsPbBr}_{3}$ PNCs from $5.9 \AA$ to $6.4 \AA$ due to iodide substitution (Fig. 2a-c). While the cuboid geometry is retained at low $\mathrm{CH}_{3} \mathrm{I}$ concentrations, clear evidence for the dissolution of the cuboid phase of the PNCs and the concurrent formation of 2DRP nanosheets is seen at higher $\mathrm{CH}_{3}$ I concentrations. Laterally-stacked 2DRP assemblies are observed upon addition of 3,000 ppbv $\mathrm{CH}_{3} \mathrm{I}$ (Fig. 2c), with the interlayer spacing of $10.0 \AA$ correlating well with that of nanosheets ${ }^{26}$. X-ray diffraction (XRD) measurements confirm that nanocuboids exist in the perovskite phase (Fig. 2e), although discrimination between the cubic and orthorhombic structures requires a more detailed structural analysis ${ }^{27}$. A progressive shift of the nanocuboid XRD peaks toward lower $2 \theta$ values occurs with increasing concentrations of $\mathrm{CH}_{3} \mathrm{I}$, in accordance with the expected lattice expansion within the iodide-rich PNCs. Moreover, consistent with HRTEM, the XRD results also indicate the formation of 2DRP at $\mathrm{CH}_{3} \mathrm{I}$ concentrations of $3,000 \mathrm{ppbv}$ and higher ${ }^{28,}{ }^{29}$. The partial transformation from 3D perovskites into 2DRP nanosheets is further supported by comparing the 2D grazing incidence 
1 wide-angle X-ray scattering (GIWAXS) measurements of the samples containing 1,000 and 26,000 ppbv $\mathrm{CH}_{3} \mathrm{I}$ (Fig. 2f, g) ${ }^{30,31}$.
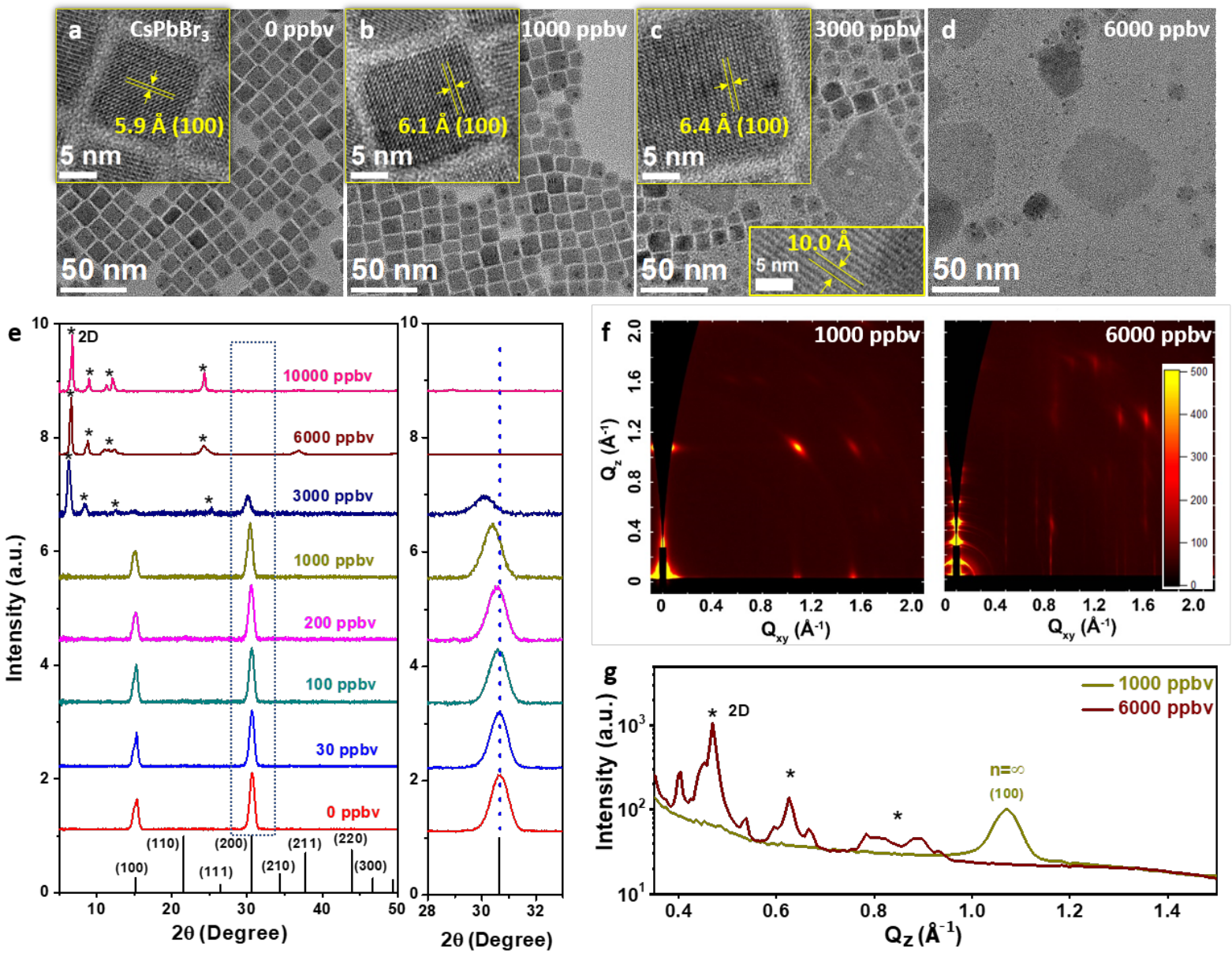

Fig. 2 | Changes to morphology and crystal structure of $\mathrm{CsPbBr}_{3} \mathrm{PNCs}$ exposed to varying

amounts of $\mathbf{C H}_{3}$ I. a, TEM images of pristine PNCs, showing a cuboid shape. b, $\mathbf{c}$, TEM images of PNCs after reaction with 1,000 ppbv and 3,000 ppbv solutions of $\mathrm{CH}_{3} \mathrm{I}$. d, TEM images of PNCs on addition of 6,000 ppbv $\mathrm{CH}_{3} \mathrm{I}$ showing almost complete loss of the cuboid PNCs, with the remaining phase comprising 2DRP nanosheets. e, XRD patterns of PNCs exposed to various $\mathrm{CH}_{3} \mathrm{I}$ amounts, which showed standard $\mathrm{CsPbBr}_{3}$ cuboid signal at both $15.2^{\circ}(100)$ and $30.6^{\circ}(200)$ for the pristine sample. $\mathbf{f}$, GIWAXS images for PNCs corresponding to $1,000 \mathrm{ppbv}$ and 6,000 ppbv $\mathrm{CH}_{3} \mathrm{I}$, respectively. $\mathbf{g}$, 1D GIWAXS profile extracted from 2D scattering patterns.

The structural transformation of the $\mathrm{CsPbBr}_{3}$ PNCs involves a complex halide exchange process. It has been suggested that this occurs through a reaction-limited exchange 
1 at the surface of the PNCs, with subsequent fast ionic diffusion occurring within the nanocrystal

2 lattice ${ }^{32}$. The spectroscopic properties of the PNCs reflect this complexity. The changes in the

3 PL emission colour occur gradually and over multiple stages of halide exchange as a function

4 of $\mathrm{CH}_{3} \mathrm{I}$ concentration (Fig. 3, S5). These stages can be categorised first according to their

5 relative PL intensity changes (Fig. 3a), with a slight enhancement of the emission (stage A)

6 followed by a rapid decrease (stage B) and then a gradual transition towards nearly complete

7 quenching (stage $\boldsymbol{C}$ ) being observed. Further inspection of the temporal changes in the UV-

8 visible absorption and PL emission within these stages reveals vastly different dynamics. UV-

9 visible absorption properties are retained in stage $\boldsymbol{A}$ (Fig. S5a, b), whereas the PL emission

10 exhibits a slight redshift and enhanced intensity (ca.10\%) after an induction period of a few

11 seconds (Fig. 3b). This behaviour is indicative of a surface-trap filling effect ${ }^{33,34}$. During stage

$12 \boldsymbol{B}$ the UV-visible absorption spectrum of the PNCs remains unchanged (Fig. S5c, d), while the

PL emission initially vanishes and then partly recovers at a red-shifted wavelength (Fig. 3c).

Finally in stage $\boldsymbol{C}$ the cuboid PNCs undergo rapid dissolution and re-nucleation as 2DRP nanosheets (Fig. S5e, f), thus resulting in a largely suppressed PL emission (Fig. 3d). For completeness, to understand the role of excess OLA across these stages, control experiments were conducted using equivalent OLA additions but without any $\mathrm{CH}_{3} \mathrm{I}$ (Fig. 3a); only a slight PL emission intensity enhancement, with no change in the peak position (see Fig. S1), was observed at the higher OLA concentrations. This demonstrates that the spectroscopic changes across the three identified stages are directly related to the alkyl ammonium iodide species generated in solution and not to the residual OLA. A schematic depiction of the structural transformation across these stages is presented in Fig. $\mathbf{3 b}$-d. 

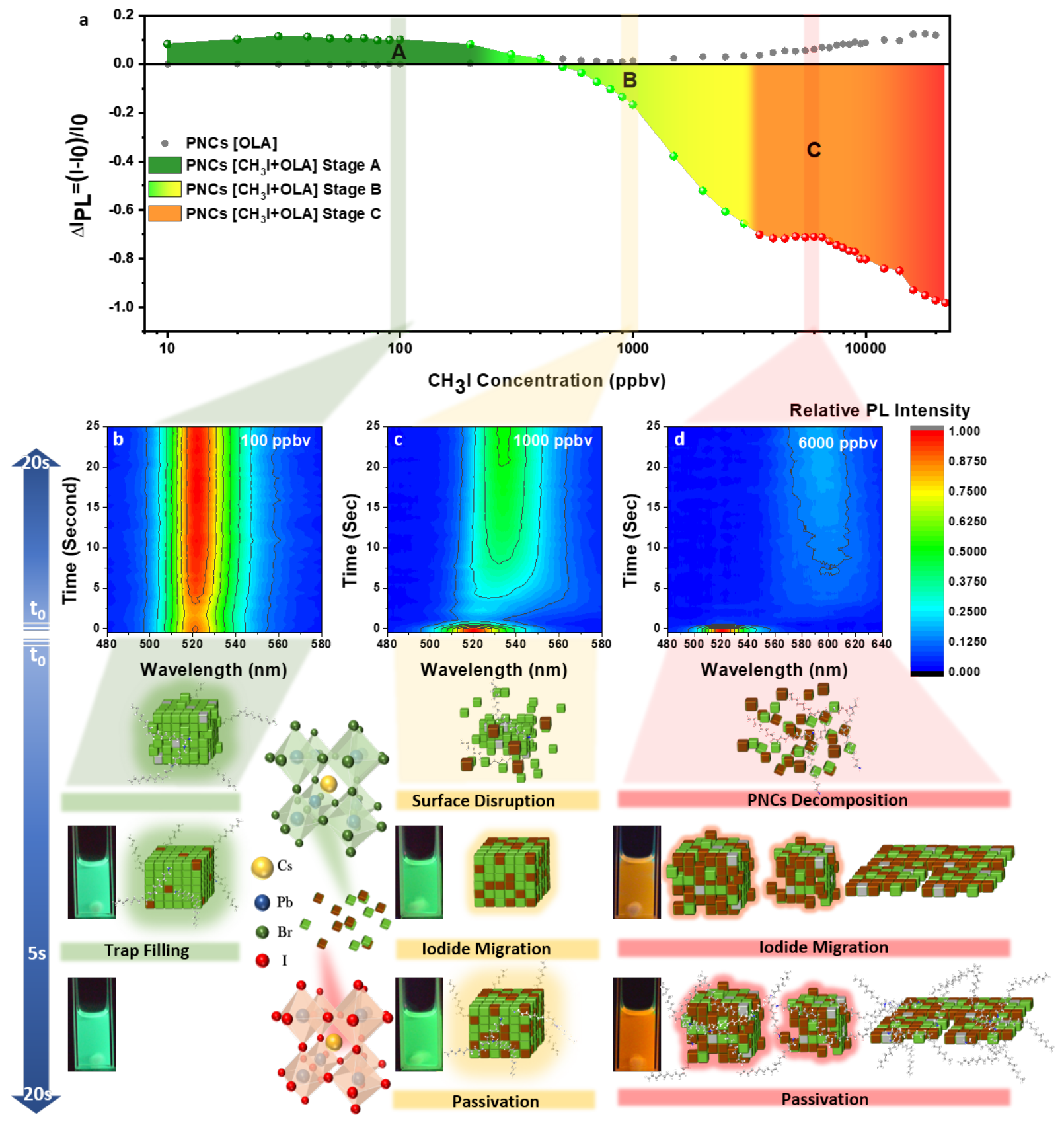

Fig. 3 | Integrated steady-state photoluminescence and millisecond time-dependent emission spectra. a, Integrated steady-state PL intensity difference displayed as a function of $\mathrm{CH}_{3} \mathrm{I}$ concentration, which explored three different stages, stage $A\left(\mathrm{CH}_{3} \mathrm{I} \leqslant 100 \mathrm{ppbv}\right)$, stage $B\left(100 \mathrm{ppbv}<\mathrm{CH}_{3} \mathrm{I} \leqslant 3000\right.$ ppbv) and stage $C\left(\mathrm{CH}_{3} \mathrm{I}>3000\right.$ ppbv). b, $\mathbf{c}, \mathbf{d}$, Time-dependent emission spectra of $\mathrm{CsPbBr}_{3} \mathrm{PNCs}$ at

$6 \mathrm{CH}_{3} \mathrm{I}$ concentration of $100 \mathrm{ppbv}, 1,000 \mathrm{ppbv}$, and 6,000 ppbv, respectively. The PNC emission images under $365 \mathrm{~nm} \mathrm{UV} \mathrm{light} \mathrm{at} 5$ and $20 \mathrm{sec}$, and the schemes of structural transformation are shown in the

8 lower panels of $\mathbf{b}, \mathbf{c}, \mathbf{d}$. It is found that the OLA-reacted $\mathrm{CH}_{3} \mathrm{I}$ in Stage $A\left(\mathrm{CH}_{3} \mathrm{I} \leqslant 100\right.$ ppbv) acts as a 
1 trap passivant, while at higher $\mathrm{CH}_{3} \mathrm{I}$ concentrations it causes the PNCs to undergo i) surface

2 disruption/PNCs decomposition, ii) iodide migration and iii) surface passivation processes in Stage B

To further correlate the ensuing structural and spectroscopic properties, the radiative relaxation dynamics of the PNCs were studied using time-resolved photoluminescence (TRPL) measurements. The PL excitation spectra in Fig. S6a suggest that, in both stage $\boldsymbol{B}$ and stage $\boldsymbol{C}$, energy transfer occurs from small n-value 2DRP nanosheets to nanocubes, consistent with previous reports ${ }^{35,36}$. The absence of PL from the 2DRP phases suggests that this energy transfer results in efficient quenching of PL from the 2DRP phases, and for this reason the PL from the nanocubes only is considered here. Analysis of the TRPL decay curves using an empirical bi-exponential function yields average PL lifetimes that show a gradual increase with increasing $\mathrm{CH}_{3} \mathrm{I}$ concentrations (Error! Reference source not found.). This is consistent with the fact that iodide- based cuboid PNCs exhibit longer radiative lifetimes than their bromide analogues (Error! Reference source not found.a) ${ }^{37,}{ }^{38}$. An accelerated slow decay in stage $\boldsymbol{A}$ suggests a reduction in the number of shallow states due to trap filling ${ }^{39,40}$. Meanwhile, the progressive increase in the lifetime of this component and its overall magnitude across stages $\boldsymbol{B}$ and $\boldsymbol{C}$ are indicative of more defective cuboid PNCs being generated (Error! Reference source not found.b, c). This confirms that the structural transformation across these stages yielding lower quality cuboid PNCs.

The chemical and structural transformations across these three stages are evidently dependent on the interaction between the oleylammonium iodide species in solution and the PNC nanocrystals. To explore this dependence, the changes in the PL redshift as a function of the PNC concentration are plotted in Error! Reference source not found.d. The results can be readily described by a semi-empirical formalism that considers the concentration of PNCs (denoted as [PNC]) as a fitting parameter (see SI-Discussion). Collectively, the results show 
1 that tuning the [PNC] provides a facile way to modulate the sensitivity window for $\mathrm{CH}_{3} \mathrm{I}$

2 detection, with lower concentrations yielding higher sensitivity factors. Given that the practical

3 range for [PNC] is $\sim 1 \mathrm{nM}$ to $\sim 1 \mathrm{mM}$, the achievable linear sensitivity window for detection of

$4 \quad \mathrm{CH}_{3} \mathrm{I}$, through [PNC] modifications alone, is $\sim 10 \mathrm{ppbv}$ to $\sim 10,000 \mathrm{ppmv}$.

5 To gauge the practical limit of the thermal response window for this sensor system, the

6 PL emission characteristics were measured between $5{ }^{\circ} \mathrm{C}$ and $45^{\circ} \mathrm{C}$ for 1,000 and 6,000 ppbv

$7 \quad \mathrm{CH}_{3} \mathrm{I}$ concentrations as representative samples (Error! Reference source not found.e-g, S8). It

8 was found that the response time is slightly longer at lower temperatures for the lower $\mathrm{CH}_{3} \mathrm{I}$

9 concentration sample, whereas temperature had little effect on response time at the higher $\mathrm{CH}_{3} \mathrm{I}$

10 concentration. Apart from slight differences in initial reaction dynamics, after a 20 seconds

11 stabilization period the PL redshifts are consistent to within $\pm 3.4 \%$ at temperatures between

$1215{ }^{\circ} \mathrm{C}$ and $35^{\circ} \mathrm{C}$ (Error! Reference source not found.g). This provides a practical temperature range for the reliable operation of a sensor platform based on this approach. 

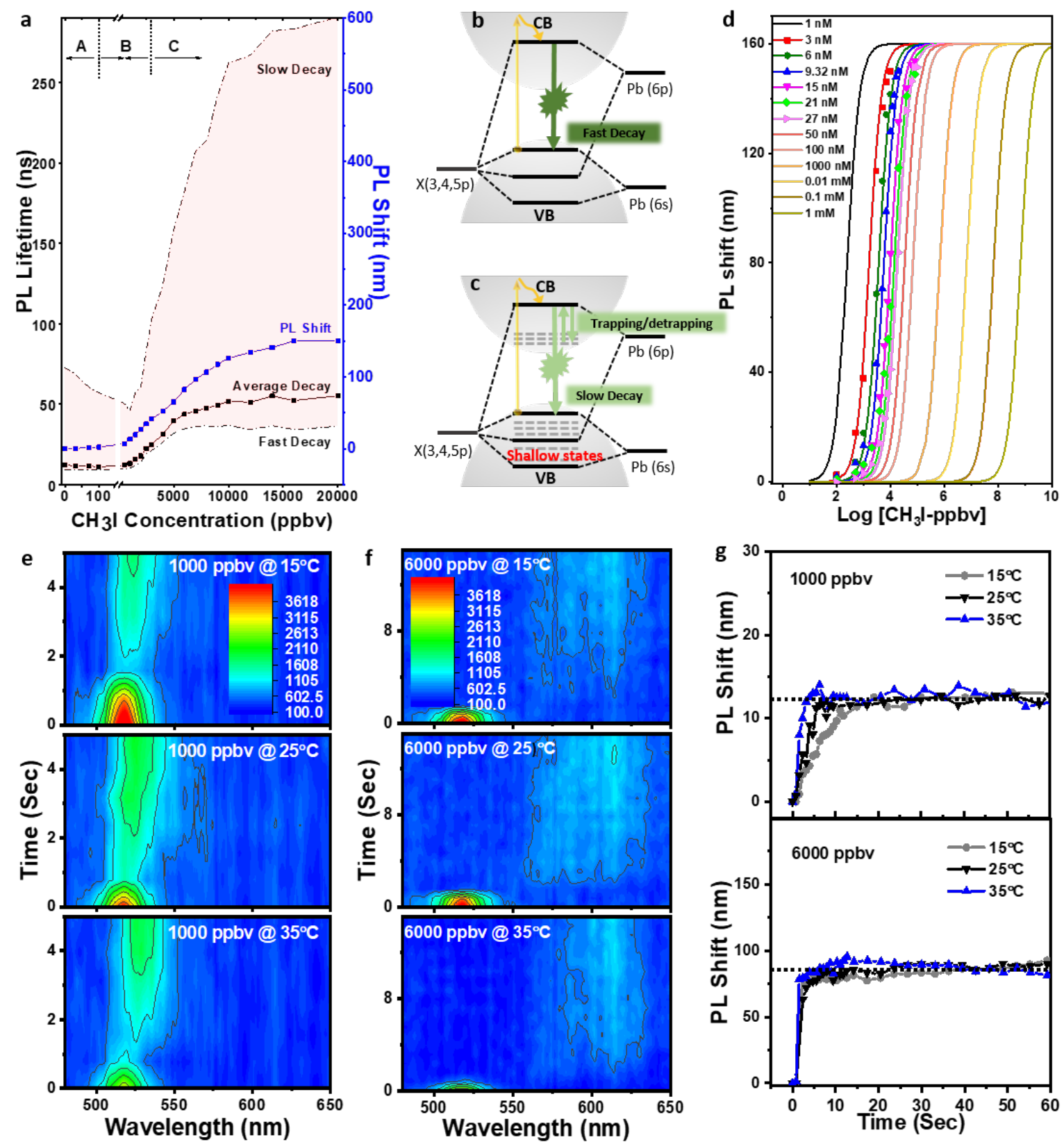

Fig. 4 | Radiative relaxation dynamics as measured by time-resolved photoluminescence (TRPL) measurements, and reliability studies under different $\mathrm{CsPbBr}_{3}$ concentrations or temperatures.

4 a, Quantitative values of slow, fast, and average PL decay as calculated from the bi-exponential fitting

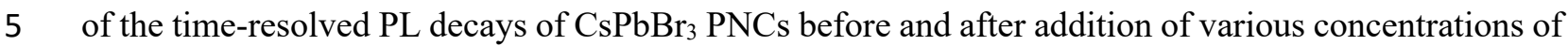

$6 \mathrm{CH}_{3} \mathrm{I}$, and corresponding PL redshifts. b, The radiative dynamic processes in ideal PNCs without

7 vacancies, where the photon is absorbed and quickly converted to prompt PL ("fast decay"). c, The

8 radiative dynamic processes in PNCs with vacancy defects, which generate shallow states inside the 
1 conduction and valance bands. The delayed PL signal is generated due to part of the excited electrons

2 traveling between shallow states, namely trapping/de-trapping, appears as a "slow decay" component.

3 d, The simulated PL redshifts (lines) of PNCs as a function of $\mathrm{CH}_{3} \mathrm{I}$ concentration, with the coloured symbols corresponding to experimental data. e, f, Time-dependent PL emission spectra of the sensor system at 15,25 , and $35^{\circ} \mathrm{C}$ at a $\mathrm{CH}_{3} \mathrm{I}$ concentration of $1,000 \mathrm{ppbv}$ and 6,000 ppbv, respectively. $\mathbf{g}$, Relative PL redshift at 15,25 , and $35^{\circ} \mathrm{C}$ after adding $\mathrm{CH}_{3} \mathrm{I}$ at a concentration of 1,000 or 6,000 ppbv, with average fluctuations $\leq 3.5 \%$ and $\leq 3.4 \%$, respectively, after $20 \mathrm{~s}$ at 15,25 , and $35^{\circ} \mathrm{C}$.

The selectivity of the $\mathrm{PNC}$-oleylamine for $\mathrm{CH}_{3} \mathrm{I}$ system was measured using a range of organoiodine compounds including iodomethane, iodoethane, iodopentane, iodohexane, iodotoluene, methyammonium iodide (MAI), formamidinium iodide (FAI), n-butylammonium iodide (n-BAI) and tetrabutylammonium iodide (TBAI) after pre-treating with OLA (see Error! Reference source not found.a, b). Negligible changes to the PL emission peak wavelength were observed for all tested organoiodines, apart from $\mathrm{CH}_{3} \mathrm{I}$. This demonstrates the high degree of selectivity of this indicator system for $\mathrm{CH}_{3} \mathrm{I}$, which can be understood by considering the dramatic differences in reaction rates of different organoiodines towards nucleophilic substitution. For a typical $\mathrm{SN}_{2}$ reaction involving iodoalkanes, the reaction rate $(\boldsymbol{R})$, given by $\boldsymbol{R}=\boldsymbol{k}[\mathbf{N u}:]\left[\mathbf{R}_{\mathbf{3}} \mathbf{C}: \mathbf{I}\right]$, is dependent on the rate constant $(\boldsymbol{k})$, the concentrations of the nucleophile (i.e. OLA in our case) and the specific organoiodine compound. Among the organoiodine derivatives studied here, $\mathrm{CH}_{3} \mathrm{I}$ has a $\boldsymbol{k}$ value that is more than 30 times larger than the others $(\text { Fig. S10 })^{20,21}$. This facilitates much faster overall dynamics, which leads to inherent selectivity for $\mathrm{CH}_{3} \mathrm{I}$ in the $\mathrm{CsPbBr}_{3} \mathrm{PNC}$ system (Fig. S11). Furthermore, this finding validates the need to generate halide salts to induce the rapid transformation of the PNCs into anion exchange analogues.

Importantly, the chemical nature of the alkyl ammonium halide salt also impacts the extent of the exchange process. This is evident from the substantially smaller spectral changes 
1 (Error! Reference source not found.a-b, S12a) and the slower exchange dynamics (Fig. S12b)

2 observed for the various ammonium iodide salts compared to those for an equivalent

3 concentration of $\mathrm{CH}_{3} \mathrm{I}$ reacted with OLA. When the PL peak changes are compared to the ratio

4 of total iodide added vs the parent bromide concentration (as determined from the [PNC]), a

5 universal curve is found for the individual iodide species. This confirms that (i) the

6 transformation mechanism in these systems is primarily dependent on the ratio of added

7 iodide/parent bromide; (ii) the different iodide sources have varied PL change characteristics;

8 and (iii) the OLA-activated $\mathrm{CH}_{3} \mathrm{I}$ provides the greatest sensitivity across the tested iodine

9 sources evaluated in the present work.

10 To provide further thermodynamic insights into these transformation trends, we

11 explored the interactions energies between $\mathrm{CH}_{3} \mathrm{I}$, OLAI and MAI with the PNC surface using first-principles density functional theory (DFT) calculations (Table S2 \& Error! Reference

source not found.c, d). The predicted positive surface interaction energy $\left(\boldsymbol{\Delta} \boldsymbol{E}_{\boldsymbol{S I}}\right)$ between $\mathrm{CH}_{3} \mathrm{I}$ and PNCs reveals a thermodynamically unfavourable chemical interaction. On the other hand, taking MAI and OLAI as representatives, the large negative surface interaction energies demonstrate that these alkyl ammonium salts favourably interact with the PNC surface. We have further investigated these potential chemical reaction pathways (Table S2 \& Fig. S13). The reaction enthalpy of pure $\mathrm{I} / \mathrm{Br}$ exchange $\left(\Delta \boldsymbol{E}_{\boldsymbol{r} e 3}\right)$ is much lower than that of just $\mathrm{Cs}$ substitution by alkyl ammonium $\left(\Delta \boldsymbol{E}_{\boldsymbol{r} e 1}\right)$ for both MAI and OLAI, indicating that halide exchange could happen spontaneously prior to other processes on the native surface of $\mathrm{CsPbBr}$. Nonetheless, this advantage diminishes when $25 \%$ pre-existing surface passivation with OLA is taken into account. It can be seen that $\mathrm{OLA}^{+}$substitution becomes the rate limiting step for halide migration in passivated PNCs (Fig. S13), which is also consistent with the previous optical discussion in Fig. 3. On the whole, the larger negative reaction energies of $\mathrm{OLA}^{+}$ compared to the shorter alkyl ammoniums, such as $\mathrm{MA}^{+}\left(\boldsymbol{\Delta} \boldsymbol{E}_{\boldsymbol{r} e 1}\right.$ and $\boldsymbol{\Delta} \boldsymbol{E}_{\boldsymbol{r} \boldsymbol{e} \text {, }}$, see Table $\left.\mathbf{S 2}\right)$, 
2 migration within the PNCs.

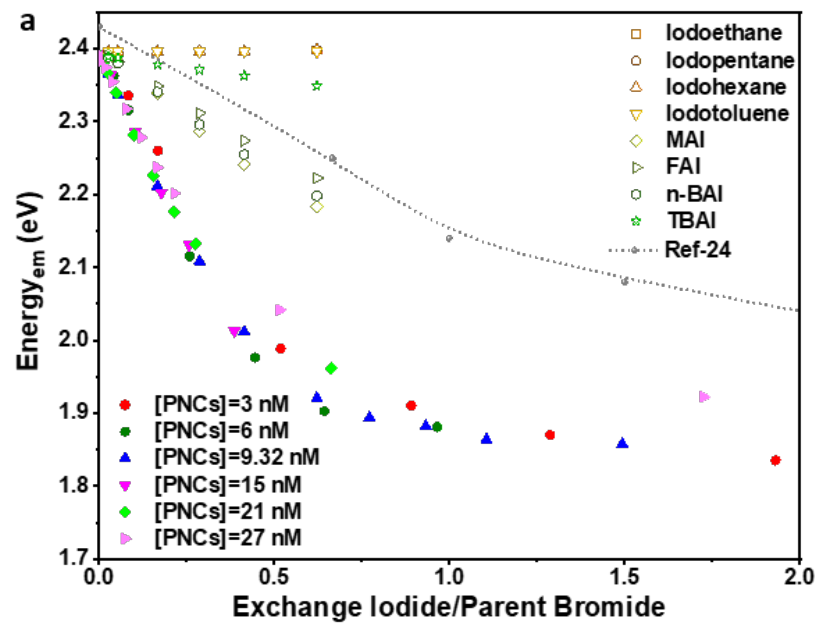

b

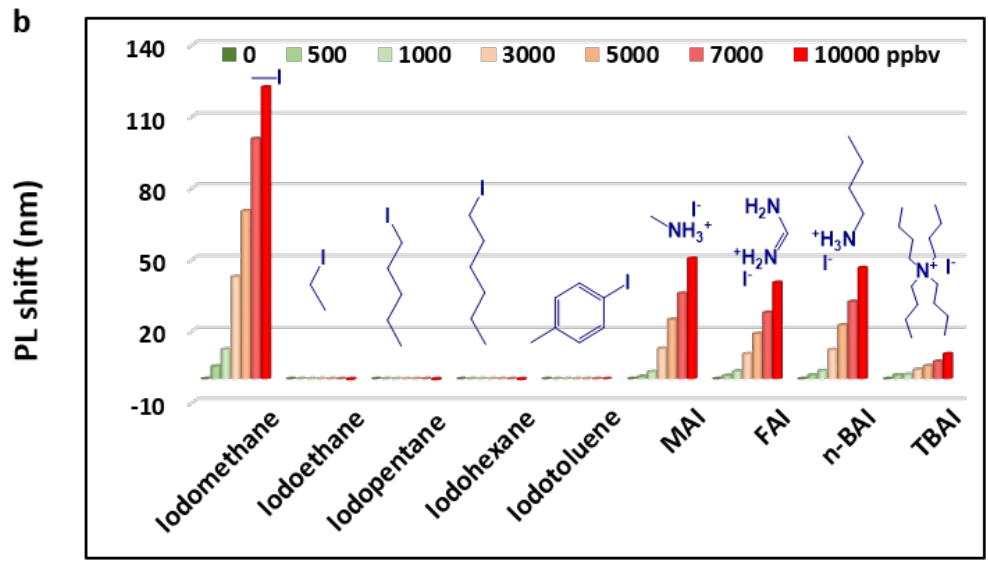

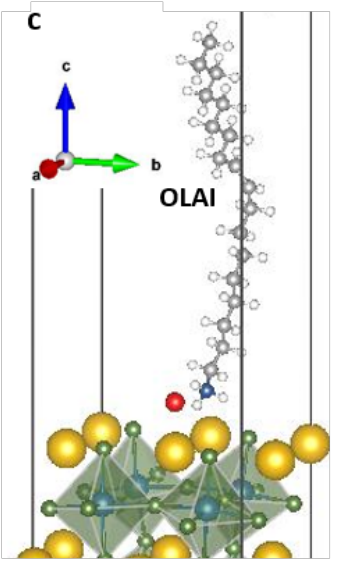
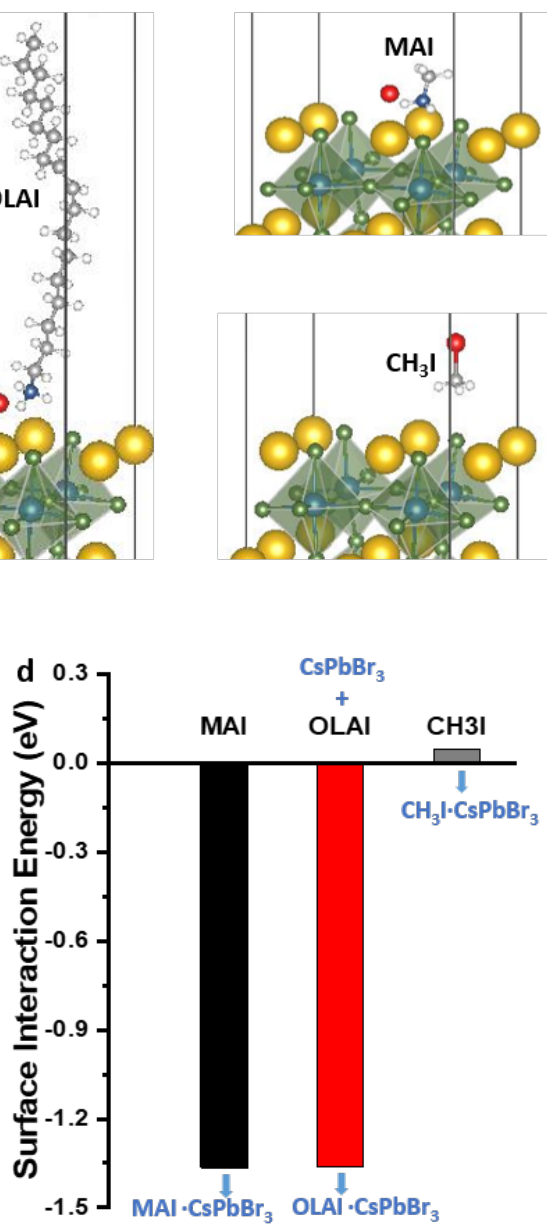

Fig. 5 | Selectivity and sensitivity studies of the $\mathrm{CsPbBr}_{3}$ nanocrystals indicator. a, Change

in the photoluminescence peak energy of the PNCs as a function of the total introduced halide species $\left(\mathrm{CH}_{3} \mathrm{I}\right.$ or anotherr other exchange halide species) relative to the starting bromide concentration within the PNC sensor solution. The energy variation response to treated $\mathrm{CH}_{3} \mathrm{I}$ is independent on the original concentration of PNCs $([\mathrm{PNC}]=3,6,9.32,15,21,27 \mathrm{nM})$. However,

9 the response is distinctly different to that of other iodide containing analogues (e.g. iodopentane, iodoethane, iodohexane, iodotoluene, methyammonium iodide (MAI), formamidinium iodide (FAI), n-butylammonium iodide (n-BAI) and tetrabutylammonium iodide (TBAI)). The dotted grey trendline is derived from literature values of the energy difference at various ratios of $\mathrm{CsPbI}_{3}: \mathrm{CsPbBr}_{3}{ }^{24}$. b, PL redshifts of PNCs with OLA-treated equimolar organoiodine 
1 analytes in IPA at various concentrations. Iodoethane, iodopentane, iodohexane, and

2 iodotoluene impart no change to the emission colour of $\mathrm{CsPbr}_{3} \mathrm{PNCs}$ due to the lack of iodide

3 formation. Meanwhile, other alkyl ammonium iodide salts, reacted less efficiently than OLA-

4 treated $\mathrm{CH}_{3} \mathrm{I}$. c, d, Schematic representation and histogram of the surface interaction energies

5 of OLAI, MAI or $\mathrm{CH}_{3} \mathrm{I}$ onto the native $\mathrm{CsPbBr}_{3}$ surface. The atomistic representations are

6 obtained after a complete relaxation of the slab models with CsBr-terminated surface using

7 first principles density functional theory (DFT) methods.

\section{Conclusion}

Metal halide perovskite nanocrystals have previously been used as optical sensors due to their attractive selectivity towards metal ions, such as copper and mercury ${ }^{41,42}$. In this work we have extended their versatility to the detection of widely used organohalides by developing a spectroscopic sensor suitable for the highly sensitive and selective detection of $\mathrm{CH}_{3} \mathrm{I}$ based on distinct changes in the $\mathrm{PL}$ of $\mathrm{CsPbr}_{3} \mathrm{PNCs}$. The transduction mechanism involves an initial methylation reaction between the $\mathrm{CH}_{3} \mathrm{I}$ and an aliphatic amine, yielding an iodide-containing ammonium salt. The liberated iodide anion undergoes lattice exchange with bromide anion, which in turn leads to structural reconfiguration of the PNCs. The ensuing changes in the PL properties provide a linear spectroscopic response and enable quantitative detection of $\mathrm{CH}_{3} \mathrm{I}$ between $100 \mathrm{ppbv}$ to $10,000 \mathrm{ppbv}$, with a limit of detection of $30 \pm 10 \mathrm{ppbv}$ and a response time of $5 \mathrm{~s}$ at room temperature. Furthermore, the significant differences in the methylation rate of aliphatic amines by $\mathrm{CH}_{3} \mathrm{I}$ compared with other organoiodine enables excellent selectivity to be achieved. This study highlights the potential applications of metal halide perovskites in other fields beyond conventional optoelectronics. In particular the nanoscale chemistry of the lead halide based perovskites opens up a unique pathway towards their application as rapid, selective and sensitive chemical detectors. 


\section{Acknowledgements}

4 This research was supported financially by the Australia Research Council funded Centre of

5 Excellence in Exciton Science (Grant No. CE170100026). The authors acknowledge the 6 technical contributions of Australian Defence Science and Technology Group (DSTG),

7 particularly the assistance and advice of Dr. Genevieve H. Dennison. W. H. acknowledges an

8 ACAP fellowship supported by the Australian Government through the Australian Renewable

9 Energy Agency (ARENA). The authors acknowledge use of the facilities and the assistance of

10 Dr. Jisheng Ma and Mr. James Griffith at the Monash X-ray Platform. M. W. and N. M. gratefully acknowledge the support from the Australian Research Council (DP166103661) and thank Monash Computing Cluster, National Computing Infrastructure and Pawsey Supercomputing Facility for the use of computational facilities. Authors also acknowledge use of the facilities at Monash Centre for Electron Microscopy (MCEM). In addition, this work was performed in part at the SAXS/WAXS beamline at the Australian Synchrotron, part of ANSTO.

\section{Author contributions}

W. Y. and J. J. J. conceived and designed the experiments, under the technical support from P. M., and W. W. H. W.. W. Y., H. L. and B. T. performed the experiments. W. Y., H. L, and J. J. analysed and constructed the results. W. H. and C. R. M. performed the GIWAXS measurement. A. D. S. performed the time-resolved photoluminescence experiments. A. S. R. C. helped with the GC-MS and NMR measurements and analysis. M. W. and N. M. performed first principles DFT calculations. W. Y., A. S. R. C., and J. J. J. mainly worked on the manuscript, with all the authors contributing to the drafting and correction.

\section{Competing Interests}


1 The authors declare no competing interests.

\section{Additional information}

3 Supplementary information available for this paper includes the synthesis of $\mathrm{CsPbI}_{3}$; GC-MS;

$4 \quad{ }^{1} \mathrm{H}-\mathrm{NMR}$; TRPL fitting table; time-dependent absorption spectra, size distribution and

5 temperature-dependent $\mathrm{PL} / \mathrm{UV}$-visible absorption of $\mathrm{CsPbBr}_{3}$ under various $\mathrm{CH}_{3} \mathrm{I}$

6 concentrations; selectivity/efficiency optical analysis; and the additional detection of various

7 bromide sources by $\mathrm{CsPbI}_{3} \mathrm{PNCs}$

8

9

\section{Methods:}

Materials and chemicals. Lead(II) bromide $\left(\mathrm{PbBr}_{2}, 99.999 \%\right.$ trace metals basis), cesium carbonate $\left(\mathrm{Cs}_{2} \mathrm{CO}_{3}, 99.995 \%\right.$ trace metals basis), oleylamine (OLA, technical grade, $70 \%$ ), diisooctylphosphinic acid (DPA, technical grade, 90\%), 1-octadecene (ODE, technical grade, $90 \%)$, iodomethane $\left(\mathrm{CH}_{3} \mathrm{I}, 99.5 \%\right.$, copper as stabilizer $)$, iodoethane $\left(\mathrm{CH}_{3} \mathrm{CH}_{2} \mathrm{I}, 99 \%\right.$, copper as stabilizer), iodopentane $\left(\mathrm{CH}_{3}\left(\mathrm{CH}_{2}\right)_{3} \mathrm{CH}_{2} \mathrm{I}, 98 \%\right.$, copper as stabilizer), 1-iodohexane (98+\%), 2iodotoluene (98\%), n-butylammonium iodide (n-BAI, 98\%), and tetrabutylammonium iodide (TBAI, $\geqslant 99.0 \%$ ) were from Sigma-Aldrich. Toluene (99.5\%) and isopropanol (IPA, 99.5\%) were from Merck. Methylammonium iodide (MAI, 99\%), and formamidinium iodide (FAI, 99\%) were from Dyenamo. All chemicals were used without further purification.

Preparation of Cs-DPA. $\mathrm{Cs}_{2} \mathrm{CO}_{3}(100 \mathrm{mg}, 0.31 \mathrm{mmol})$ was added to a $25 \mathrm{ml}$ 2-neck flask containing ODE $(5 \mathrm{ml})$ and DPA $(0.5 \mathrm{ml})$, and heated at $120^{\circ} \mathrm{C}$ under vacuum (ca. 2 mbar) for 30 min to remove residual water. The temperature was then raised to $150{ }^{\circ} \mathrm{C}$ under $\mathrm{N}_{2}$, with the temperature maintained until the complete dissolution of $\mathrm{Cs}_{2} \mathrm{CO}_{3}$. The solution was stored under $\mathrm{N}_{2}$ at room temperature, and heated to $120{ }^{\circ} \mathrm{C}$ prior to injection in subsequent reactions. Synthesis of $\mathbf{C s P b B r} 3$ PNCs. $\mathrm{PbBr}_{2}(69 \mathrm{mg}, 0.188 \mathrm{mmol})$ was added to ODE (5 ml) in a 50 ml 3-neck flask, with OLA $(0.5 \mathrm{ml})$ and DPA $(0.5 \mathrm{ml})$ then added to the suspension. The 
1 solution was then heated to $120^{\circ} \mathrm{C}$ under vacuum (ca. 2 mbar) with stirring for $30 \mathrm{~min}$ in order

2 to remove residual water and dissolve the $\mathrm{PbBr}_{2}$. Upon complete dissolution of the $\mathrm{PbBr}_{2}$, the

5 the solution was cooled in an ice bath.

6 Isolation and Purification of $\mathrm{CsPbBr}_{3}$ PNCs. The crude reaction solution was mixed with

7 IPA (crude solution:IPA $=1: 3 \mathrm{v} / \mathrm{v}$ ), resulting in the precipitation of most of the PNCs. After

centrifugation at $10,000 \mathrm{rpm}$ for $5 \mathrm{~min}$, the supernatant was discarded and the precipitated PNCs were redispersed in toluene to give a solution that was colloidally stable over an extended period.

Preparation of PNC Sensor and Analyte Solutions. The perovskite NC sensor solution was prepared by diluting the washed perovskite dispersion with toluene to $3 \mathrm{ml}$. The concentration of PNCs was adjusted and maintained at about $9.32 \pm 0.15 \mathrm{nM}$, which was calculated by the absorption and the relative molar extinction coefficient as determined in the study of Maes et $\mathrm{al}^{43}$. A stock solution of $\mathrm{CH}_{3} \mathrm{I}$ was prepared by adding $\mathrm{CH}_{3} \mathrm{I}(10 \mu \mathrm{L}, \sim 30.9 \mu \mathrm{M})$ toluene or IPA $(1 \mathrm{ml})$, followed by the addition of OLA (ca. $90 \mu \mathrm{M}$ ). This solution was then diluted 120 -fold and a portion was added to the sensor solution to add a known concentration of $\mathrm{CH}_{3} \mathrm{I}$. Other analyte solutions were prepared using a similar method.

Characterization. Samples for powder X-ray diffraction (XRD) were prepared by dropcasting a PNC dispersion onto a glass substrate, which was then dried without heating in an ambient environment. XRD patterns were collected by a Bruker D8 Advance diffractometer equipped with a $\mathrm{Cu}-\mathrm{K} \alpha$ radiation source operated at $40 \mathrm{kV}$ and $40 \mathrm{~mA}$, and equipped with a high-speed line-position sensitive Lynxeye XE detector. Transmission electron microscopy (TEM) images of each sample on ultra-thin carbon grids were collected using a Tecnai $\mathrm{G}^{2} \mathrm{~T} 20$ TWIN (FEI Company) microscope equipped with a $\mathrm{LaB}_{6}$ electron emitter. The steady-state 
1 absorption and emission spectra were recorded with a commercial spectrometer (StellarNet)

2 equipped with a CCD camera (Silver-Nova-TEC-X2), using a halogen lamp (SL1) or $390 \mathrm{~nm}$

excitation source (SL1-LED), respectively. The relative time-course experiment spectra were collected using the same system. Time-resolved photoluminescence (TRPL) spectra were measured using an FLS 980 spectrometer (Edinburgh Instruments) with a $405 \mathrm{~nm}$ pulsed diode laser for excitation with a $500 \mathrm{kHz}$ frequency (average power up to $6.25 \mu \mathrm{W}$ ) and the maximal emission wavelength of each sample used for detection. The 2-Dimensional grazing incidence wide-angle X-ray scattering (GIWAXS) was measured at the SAXS/WAXS beamline at Australian Synchrotron ${ }^{44,45}$. The energy of the incident X-ray beam was fixed at $15 \mathrm{keV}$. The 2D scattering patterns were acquired using a Dectris Pilatus $1 \mathrm{M}$ detector with an exposure time of $1 \mathrm{~s}$ and an incident angle of $0.2^{\circ}$. GC-mass spectra were obtained with a Thermo Scientific TSQ 8000 TRACE 1310 GC mass spectrometer using electron ionization in the positive ion mode with ionization energy of $70 \mathrm{eV}$. Gas chromatography was performed on a SGE SOLGEL-1MS column $(30 \mathrm{~m} \times 0.25 \mathrm{~mm}$ ID, $0.25 \mu \mathrm{m}$ film thickness $)$, with a temperature program of $50{ }^{\circ} \mathrm{C}$ for $2 \mathrm{~min}$, then heating at $25^{\circ} \mathrm{C} / \mathrm{min}$ to $300{ }^{\circ} \mathrm{C}$ where the temperature was held for 8 minutes with a split injection, split ratio of 10 , an injector temperature of $300^{\circ} \mathrm{C}$ and the transfer line was set to $300{ }^{\circ} \mathrm{C}$. High-purity helium was used as carrier gas with a flow rate of $1 \mathrm{ml} / \mathrm{min} .{ }^{1} \mathrm{H}-\mathrm{NMR}$ measurements were performed on a Bruker Bio Spin Av400H with a 9.4 T magnet and a $5 \mathrm{~mm}$ inverse $1 \mathrm{H}-\mathrm{X}$ BBI autotuning broadband probe at a ${ }^{1} \mathrm{H}$ frequency of 400.13 MHz in toluene- $d 8$.

Computational Methods. First principles, density functional theory (DFT) calculations were carried out using the Vienna Ab Initio Simulation Package (VASP) ${ }^{46}$. The projector augmented wave $(\mathrm{PAW})^{47}$ pseudopotentials were utilized to describe core and valence electrons, and the generalized gradient approximation based on the Perdew-Burke-Ernzerhof (GGA-PBE) ${ }^{48}$ form was selected to describe electron exchange and correlation. Grimme's energy correction 
1 method was employed to correctly account for the dispersion interactions ${ }^{49}$. Various chemical

2 reactions at the $\mathrm{CsBr}$-terminated neutral surface of $\mathrm{CsPbBr}_{3}$ nanoscrystals were investigated

\section{References} (2016).

1. Ashworth, D. J., Yates, S. R., Luo, L. \& Xuan, R. Phase partitioning, retention kinetics, and leaching of fumigant methyl iodide in agricultural soils. Sci. Total Environ. 432, 122-127 (2012).

2. Tang, P. X., Nguyen, N. T. H., Lo, J. G. \& Sun, G. Colorimetric Detection of Carcinogenic Alkylating Fumigants on a Nylon 6 Nanofibrous Membrane. Part II: Self-Catalysis of 2Diethylaminoethyl-Modified Sensor Matrix for Improvement of Sensitivity. Acs Applied Materials \& Interfaces 11(14), 13632-13641 (2019).

3. Guthman, J. \& Brown, S. I will never eat another strawberry again: the biopolitics of consumercitizenship in the fight against methyl iodide in California. Agr. Human Values 33(3), 575-585

4. Guthman, J. \& Brown, S. Midas' Not-So-Golden Touch: On the Demise of Methyl Iodide as a Soil Fumigant in California. Journal of Environmental Policy \& Planning 18(3), 324-341 (2016).

5. Tang, P. X., Leung, H. T. \& Sun, G. Colorimetric Detection of Carcinogenic Alkylating Fumigants on Nylon-6 Nanofibrous Membrane. Part I: Investigation of 4-(p-Nitrobenzyl)pyridine as a "New" Sensing Agent with Ultrahigh Sensitivity. Anal. Chem. 90(24), 14593-14601 (2018).

6. Bale, C. S. E., et al. Novel measurements of atmospheric iodine species by resonance fluorescence. $J A t C$ 60(1), 51-70 (2008). 
1 7. Mekic, M., Temime-Roussel, B., Monod, A. \& Strekowski, R. S. Quantification of gas phase methyl iodide using $\mathrm{H} 3 \mathrm{O}+$ as the reagent ion in the PTR-MS technique. Int. J. Mass spectrom. 424, $10-15(2018)$.

8. Das, S., Sharma, P., Badani, P. M. \& Vatsa, R. K. Ionisation of methyl iodide clusters using nanosecond laser pulses: detection of multiply charged positive ions, negative ions and energetic electrons. Rsc Advances 5(12), 8887-8894 (2015).

9. R'Mili, B., et al. Quantification of the gas phase methyl iodide using $\mathrm{O}-2(+)$ as the reagent ion in the PTR-ToF-MS technique. Int. J. Mass spectrom. 431, 43-49 (2018).

10. Kim, T., et al. Concentration Determination of Volatile Molecular Iodine and Methyl Iodide. Bull. Korean Chem. Soc. 39(6), 824-828 (2018).

11. Wu, C., et al. Chemoselective detection of alkyl halides via an iridium(III) luminescent probe. Dyes and Pigments 159, 479-482 (2018).

12. Chen, W., et al. A pyridinium cation- $\pi$ interaction sensor for the fluorescent detection of alkyl halides. Chemical Communications 47(1), 253-255 (2011).

13. Hertzog-Ronen, C., et al. Detection and Identification of Alkylating Agents by Using a Bioinspired “Chemical Nose”. Chemistry - A European Journal 15(40), 10380-10386 (2009).

14. Kovalenko, M. V., Protesescu, L. \& Bodnarchuk, M. I. Properties and potential optoelectronic applications of lead halide perovskite nanocrystals. Science 358(6364), 745 (2017).

15. Huang, H., et al. Lead Halide Perovskite Nanocrystals in the Research Spotlight: Stability and Defect Tolerance. ACS Energy Letters 2(9), 2071-2083 (2017).

16. Schmidt, L. C., et al. Nontemplate Synthesis of CH3NH3PbBr3 Perovskite Nanoparticles. J. Am. Chem. Soc. 136(3), 850-853 (2014).

17. Shamsi, J., et al. Metal Halide Perovskite Nanocrystals: Synthesis, Post-Synthesis Modifications, and Their Optical Properties. Chem. Rev. 119(5), 3296-3348 (2019).

18. Doane, T. L., et al. Using Perovskite Nanoparticles as Halide Reservoirs in Catalysis and as Spectrochemical Probes of Ions in Solution. ACS Nano 10(6), 5864-5872 (2016).

19. Jang, D. M., et al. Reversible Halide Exchange Reaction of Organometal Trihalide Perovskite Colloidal Nanocrystals for Full-Range Band Gap Tuning. Nano Lett. 15(8), 5191-5199 (2015). 
1 20. Robert C. Neuman, J. Reactions of Haloalkanes, Alcohols, and Amines : Nucleophilic Substitution. Organic Chemistry, chapter 7, 1-51 (2013).

21. Robert C. Neuman, J. Haloalkanes, Alcohols, Ethers, and Amines. Organic Chemistry, chapter 3, $1-59$ (2013).

22. Pan, A., et al. Insight into the Ligand-Mediated Synthesis of Colloidal CsPbBr3 Perovskite Nanocrystals: The Role of Organic Acid, Base, and Cesium Precursors. ACS Nano 10(8), 79437954 (2016).

23. De Roo, J., et al. Highly Dynamic Ligand Binding and Light Absorption Coefficient of Cesium Lead Bromide Perovskite Nanocrystals. ACS Nano 10(2), 2071-2081 (2016).

24. Akkerman, Q. A., et al. Tuning the Optical Properties of Cesium Lead Halide Perovskite Nanocrystals by Anion Exchange Reactions. J. Am. Chem. Soc. 137(32), 10276-10281 (2015).

25. Shi, E., et al. Two-dimensional halide perovskite nanomaterials and heterostructures. Chem. Soc. Rev. 47(16), 6046-6072 (2018).

26. Weidman, M. C., Goodman, A. J. \& Tisdale, W. A. Colloidal Halide Perovskite Nanoplatelets: An Exciting New Class of Semiconductor Nanomaterials. Chem. Mater. 29(12), 5019-5030 (2017).

27. Brennan, M. C., Kuno, M. \& Rouvimov, S. Crystal Structure of Individual CsPbBr3 Perovskite Nanocubes. Inorg. Chem. 58(2), 1555-1560 (2019).

28. Blancon, J. C., et al. Extremely efficient internal exciton dissociation through edge states in layered 2D perovskites. Science 355(6331), 1288 (2017).

29. Yang, X., et al. Efficient green light-emitting diodes based on quasi-two-dimensional composition and phase engineered perovskite with surface passivation. Nature Communications 9(1), 570 (2018).

30. Chen, Z. M., et al. Stable Sn/Pb-Based Perovskite Solar Cells with a Coherent 2D/3D Interface. Iscience 9, 337-346 (2018).

31. Wang, Z., et al. Efficient ambient-air-stable solar cells with 2D-3D heterostructured butylammonium-caesium-formamidinium lead halide perovskites. Nature Energy 2, 17135 (2017). 
32. Koscher, B. A., et al. Surface- vs Diffusion-Limited Mechanisms of Anion Exchange in CsPbBr3 Nanocrystal Cubes Revealed through Kinetic Studies. J. Am. Chem. Soc. 138(37), 12065-12068 (2016).

33. Xu, W., et al. Iodomethane-Mediated Organometal Halide Perovskite with Record Photoluminescence Lifetime. ACS Applied Materials \& Interfaces 8(35), 23181-23189 (2016).

34. Stoeckel, M.-A., et al. Halide Perovskites: Reversible, Fast, and Wide-Range Oxygen Sensor Based on Nanostructured Organometal Halide Perovskite Adv. Mater. 29(38), 1702469 (2017).

35. Li, M., et al. Amplified Spontaneous Emission Based on 2D Ruddlesden-Popper Perovskites. Adv. Funct. Mater. 28(17), 1707006 (2018).

36. Protesescu, L., et al. Nanocrystals of Cesium Lead Halide Perovskites (CsPbX3, $\mathrm{X}=\mathrm{Cl}, \mathrm{Br}$, and I): Novel Optoelectronic Materials Showing Bright Emission with Wide Color Gamut. Nano Lett. 15(6), 3692-3696 (2015).

37. Zhang, Y., et al. Quantitative imaging of anion exchange kinetics in halide perovskites. Proceedings of the National Academy of Sciences 116(26), 12648 (2019).

38. Zhang, D., et al. Synthesis of Composition Tunable and Highly Luminescent Cesium Lead Halide Nanowires through Anion-Exchange Reactions. J. Am. Chem. Soc. 138(23), 7236-7239 (2016).

39. Chirvony, V. S., et al. Delayed Luminescence in Lead Halide Perovskite Nanocrystals. The Journal of Physical Chemistry C 121(24), 13381-13390 (2017).

40. Brandt, R. E., et al. Searching for "Defect-Tolerant" Photovoltaic Materials: Combined Theoretical and Experimental Screening. Chem. Mater. 29(11), 4667-4674 (2017).

41. Liu, Y., et al. All-inorganic $\mathrm{CsPbBr} 3$ perovskite quantum dots as a photoluminescent probe for ultrasensitive Cu2+ detection. Journal of Materials Chemistry C 6(17), 4793-4799 (2018).

42. Lu, L. Q., et al. Visual and sensitive fluorescent sensing for ultratrace mercury ions by perovskite quantum dots. Anal. Chim. Acta 986, 109-114 (2017).

43. Maes, J., et al. Light Absorption Coefficient of $\mathrm{CsPbBr} 3$ Perovskite Nanocrystals. The Journal of Physical Chemistry Letters 9(11), 3093-3097 (2018).

44. Huang, W., et al. Probing Molecular and Crystalline Orientation in Solution-Processed Perovskite Solar Cells. Adv. Funct. Mater. 25(34), 5529-5536 (2015). 
1 45. Kirby, N. M., et al. A low-background-intensity focusing small-angle X-ray scattering undulator beamline. J. Appl. Crystallogr. 46(6), 1670-1680 (2013).

46. Kresse, G. \& Furthmüller, J. Efficiency of ab-initio total energy calculations for metals and semiconductors using a plane-wave basis set. Computational Materials Science 6(1), 15-50 (1996).

47. Kresse, G. \& Joubert, D. From ultrasoft pseudopotentials to the projector augmented-wave method. Physical Review B 59(3), 1758-1775 (1999).

48. Perdew, J. P., Burke, K. \& Ernzerhof, M. Generalized Gradient Approximation Made Simple. Physical Review Letters 77(18), 3865-3868 (1996).

49. Grimme, S., Antony, J., Ehrlich, S. \& Krieg, H. A consistent and accurate ab initio parametrization of density functional dispersion correction (DFT-D) for the 94 elements H-Pu. The Journal of Chemical Physics 132(15), 154104 (2010). 


\section{University Library}

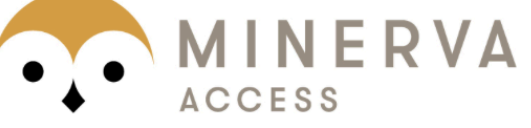

A gateway to Melbourne's research publications

Minerva Access is the Institutional Repository of The University of Melbourne

Author/s:

Yin, W;Li, H;Chesman, ASR;Tadgell, B;Scully, AD;Wang, M;Huang, W;McNeill, CR;Wong,

WWH;Medhekar, N;Mulvaney, P;Jasieniak, JJ

Title:

Detection of Halomethanes Using Cesium Lead Halide Perovskite Nanocrystals

Date:

2021-01-13

\section{Citation:}

Yin, W., Li, H., Chesman, A. S. R., Tadgell, B., Scully, A. D., Wang, M., Huang, W., McNeill, C. R., Wong, W. W. H., Medhekar, N., Mulvaney, P. \& Jasieniak, J. J. (2021). Detection of Halomethanes Using Cesium Lead Halide Perovskite Nanocrystals. ACS NANO, 15 (1), pp.1454-1464. https://doi.org/10.1021/acsnano.0c08794.

Persistent Link:

http://hdl.handle.net/11343/285512 